Sharif University of Technology
Scientia Iranica
SCIENTIA
IRAN I CA

\title{
Experimental investigation into shock waves formation and development process in transonic flow
}

\author{
M. Farahani* and A. Jaberi \\ Department of Aerospace Engineering, Sharif University of Technology, Tehran, Iran.
}

Received 11 May 2016; received in revised form 12 August 2016; accepted 8 October 2016

\section{KEYWORDS}

Shock waves

formation process;

Transonic flow;

Shadowgraphy

visualization;

Splitting of shock

waves;

Zeh shock.

\begin{abstract}
An extensive experimental investigation was performed to explore the shock waves formation and development process in transonic flow. Shadowgraph visualization technique was employed to provide visual description of the flowfield features. Based on the visualization, the formation process was categorized into two intrinsically different phases, namely, subsonic and supersonic. The characteristics of subsonic phase are well known; however, those of the supersonic one are far less studied. The supersonic phase itself is made up of two consecutive phases, namely, approaching and sweeping. The effects of each phase on the flowfield characteristics and on shaping the supersonic regime were studied in details. In order to generalize the results, three different models were tested. A special terminology is suggested by authors to ease the process description and pave a way for future studies. Above all, as the transition from transonic regime to the supersonic one is a vague concept in terms of physical reasoning, a new explanation is proposed that can be used as a criterion for distinguishing between transonic and supersonic regimes.

(C) 2017 Sharif University of Technology. All rights reserved.
\end{abstract}

\section{Introduction}

Knowledge of the flow conditions over a body in transonic and supersonic speeds is of great importance in various aspects, particularly the appearance of shock waves. These established shocks have profound effects on the aerodynamic characteristics of a vehicle and, therefore, a detailed investigation is necessary. A principal phenomenon related to shock waves is their formation and development process that takes place within transonic regime. This process has been studied by many research studies; however, there are still many aspects that require further investigation.

Broadly speaking, a shock wave occurs in supersonic flow because of two reasons: first, to turn the

\footnotetext{
*. Corresponding author. Tel.: +9821 66022731;

Fax: +982166022731

E-mail address: mfarahani@sharif.edu (M. Farahani)
}

streamlines tangent along the non-penetrating surface or/and, second, to compensate for the pressure difference between upstream and downstream and satisfy pressure boundary conditions. The former is usually seen in external flows and the latter mostly happens in nozzles and diffusers. In some supersonic wind tunnels such as the one used here, at first, the nozzle exit to throat ratio is set; then, during the startup and after the flow reaches sonic conditions at the throat, a primary normal shock appears, which separates supersonic and subsonic regions. In order to reach the determined Mach number at test section by increasing blowing power of wind tunnel engine, the normal shock moves downstream and becomes stronger. In this phase, acceleration of the flow results in the variable strength of the normal shock wave, which is the main source of unsteadiness. On the other hand, in the presence of a model in test section, some shock or expansion waves would be established over it to ensure that the flow remains in the direction of the body surface everywhere. Due to this, the strengths of 
these waves vary by acceleration of flow as they are dependent upon the upcoming normal shock. This introduces the second source of unsteadiness in this case. Therefore, the current investigation deals with the transient case of transonic regime.

The formation process can consist two consecutive phases; the first begins as the free-stream Mach number reaches its critical value and the second starts when $M_{\infty}$ passes the unity. Numerous studies have investigated the first phase of the formation process (e.g., [1-6]); establishment of lambda shock waves, interaction with boundary layer, and separation under different circumstances are the main focus of such studies. In contrast, there are only a few works regarding the case when the free-stream flow passes the sound speed. Patz [7] investigated the formation of bow waves around blunt bodies in the flow behind a mowing shock and derived stand-off distances and formation times for various blunt bodies. Davies [8] also experimentally explored the shock waves structure when a primary normal shock passed over a blunt body; further, he suggested a schematic description of the formation process, which was reproduced with very more details in this work.

In this survey, both phases are investigated and organized in details; but, since less attention has been paid to the supersonic phase, an in-depth effort is put to identify its unique characteristics. The process continues until the transonic regime ends and the supersonic regime begins. To authors' knowledge, there is no approved physical explanation to determine this transition, even though Mach number of 1.2, which is strictly a rule of thumb, is usually implemented. In this study, a physical definition is proposed to differentiate between transonic and supersonic regimes.

To follow the formation and development processes of shock waves in details, visualization methods seem to be very useful tools. In this research, shadowgraph technique is employed. Shadowgraphy is the most ubiquitous and simplest of all the optical flow diagnostics, which is also the best for imaging shock waves. It reveals shock waves clearly while deemphasizing other less abrupt flow features [9].

The supersonic phase of shock wave formation and development process is described thoroughly for the model and new phenomena and characteristics are introduced. Obviously, these results cannot be confirmed and generalized if only one case is taken under consideration. For this sake, shadowgraphs of three models are compared. Further, the effect of nose shape on the process is investigated.

Experimental studies are necessary in order to provide "benchmark" data for CFD code validation and, primarily, to understand the fluid dynamics of a phenomenon. The present study falls within the latter category. It describes the shock system formation and development, qualitatively, in an attempt to illustrate its essential features and, further, to provide a tentative basis for more rigorous investigations in future. In the following, in Section 2, wind tunnel and testing systems will be described. The results will be discussed and interpreted in Section 3, and the corresponding conclusions are summarized in Section 4.

\section{Experimental details}

All tests are conducted in a suction type Trisonic wind tunnel with a Mach number capability of 0.4-2.2. It has a test section of $60 \mathrm{~cm} \times 60 \mathrm{~cm} \times 120 \mathrm{~cm}$ and is equipped with various internal strain gauge balances, pressure transducers, high-speed camera, shadowgraph system, etc. A detailed description of the wind tunnel can be found in [10].

Three models with different configurations and different nose shapes are provided for tests. The first model is an axisymmetric body with a blunt nose and a maximum diameter of $64.3 \mathrm{~mm}$. The detailed description of the model geometry and its nomenclature is given in Figure 1(a). This geometrical nomenclature is used to ease the interpretation of flow characteristics. The second model, the ogive one, is a long axisymmetric body and has a fineness ratio of 2.5 equipped with a circular-arc, ogival nose tangent to a cylindrical after-body with $L / d=15$ (Figure $1(\mathrm{~b})$ ). Moreover, in order to study the effects of cross section changes on the formation process, a belt with inclination angle of 5 degrees is installed on its cylindrical section. In addition, a guitar wire with a diameter of $0.9 \mathrm{~mm}$ is installed at $x / d=6.25$ to induce disturbances into the flowfield. These two cases are very helpful to survey the supersonic phase of the shock formation process.

The third model, namely, the inlet model, is a supersonic axisymmetric external compression model, as shown in Figure 1(c), of which the main model parts are specified. The main parts include an external cowl that produces flow compression and is connected to an axisymmetric subsonic diffuser, consisting of a cowl and a spike. The selection of these models is based on their different nose shapes, which mostly affect the characteristics of supersonic phase. In effect, blunt nose, sharp nose, and inlet nose models cover nearly all possibilities that can happen in the shock formation process.

Tests are performed at free-stream Mach numbers ranging from 0.6 to 2.0. For recording the shadowgraphs, a CASIO EX-F1 digital video camera is used, which provides high-speed imaging. It can record 300 frames per second with a resolution of $512 \times 384$ pixels and is capable of $1200 \mathrm{fps}$ at further reduced image size.

\section{Results and discussion}

Based on the free-stream Mach number, shock wave 


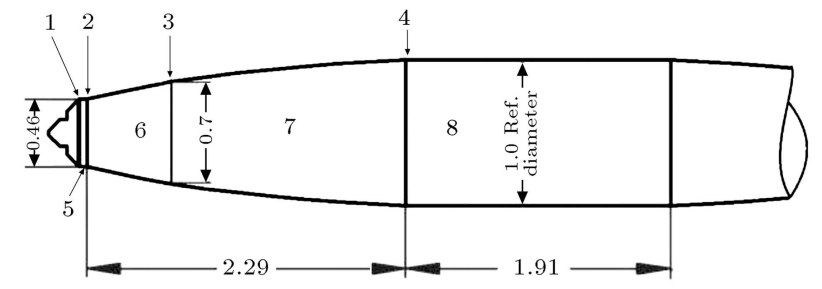

1- Nose-belt junction (junction 1) 2- Belt-cone junction (junction 2)

3- Cone-parabola junction (junction 3 )

4- Parabola-cylinder junction (junction 4)

5- Belt region 6- Conical region 7- Parabolic region 8- Cylindrical region

(a)
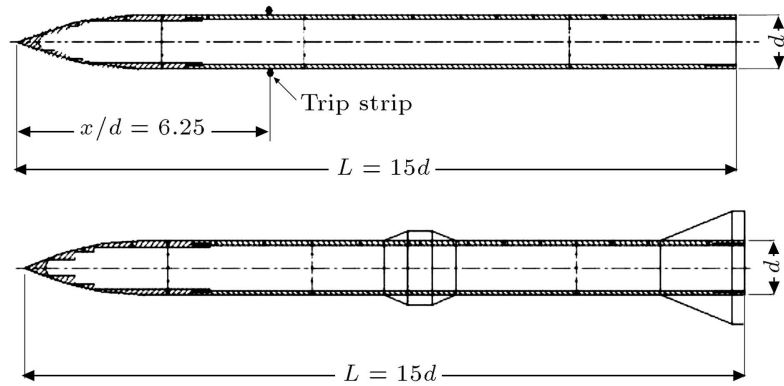

(b)

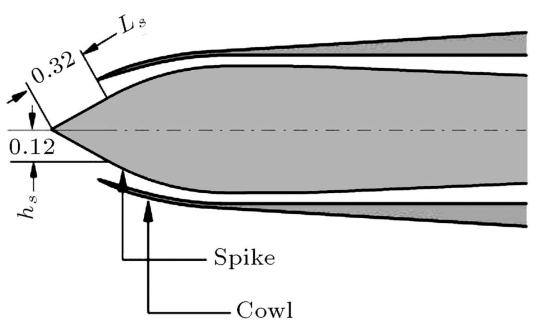

(c)

Figure 1. Schematic of different models used (all linear dimensions in calibers): (a) Model A: its geometry, and nomenclature, (b) model B: Ogive model Perturbed wire (up), belt-installed (down), and (c) model C: inlet model.

formation and development process have been categorized into two consecutive phases. The first phase depicts the formation process when the free-stream Mach number is lower than unity (subsonic phase), while at the second phase it increases to $M_{\infty}>1$ (supersonic phase). The second phase has more complexities and, hence, reveals more special behaviors. (Note: subsonic and supersonic phases are just two steps of transonic regime and must not be confused with subsonic and supersonic regimes). In the following, the principal characteristics of the process will be explored via detailed description of the shadowgraphs of model A and, then, the results will be compared with ogive and inlet models.

\subsection{Subsonic phase}

Figure 2 shows a series of shadowgraphs illustrating the flowfield around model A for the subsonic phase of shock formations. This figure consists of 12 steps and
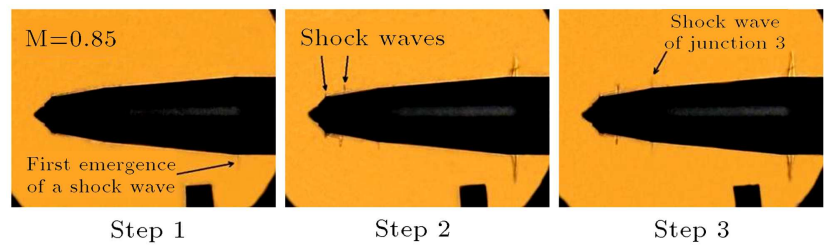

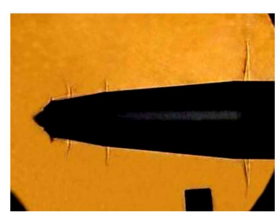

Step 4

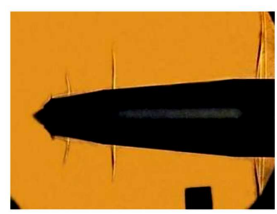

Step 7

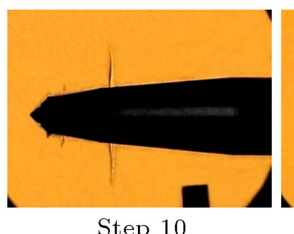

Step 10

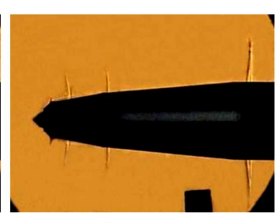

Step 5

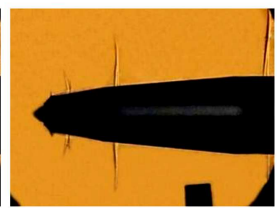

Step 8

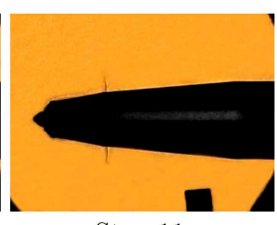

Step 11

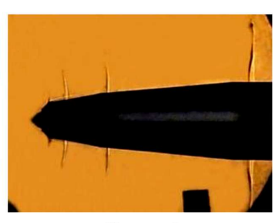

Step 6

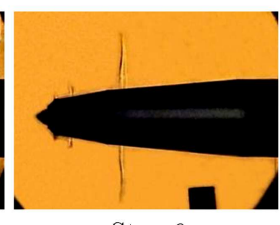

Step 9

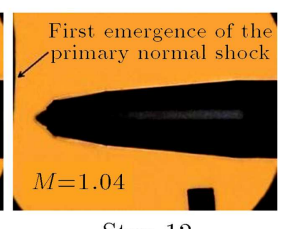

Step 12
Figure 2. Shadowgraphic visualization of the subsonic phase around model A.

begins with the first appearance of a shock wave over the model (critical free-stream Mach number) in Step 1 and ends with the first appearance of the shock in front of the model (supersonic free-stream Mach number) in Step 12.

At the first step, the flow expansion after the parabola-cylinder junction produces a locally supersonic flow. This supersonic flow then becomes subsonic via a weak normal shock right after junction 4 (as seen in Figure 1). Even though this normal shock is very inconspicuous, its first advent is constituted as the first step of shock wave formation process.

At Step 2, new shocks appear at junction 1 and on the conical region. The small normal shock forming after junction 1 is very weak and, according to the shadowgraphs, its strength does not change significantly by increasing the free-stream Mach number. The formed shock on the conical region is placed right in the separated flow, though it does not affect the separation pattern. More importantly, the primary normal shock at junction 4 is shaped like a lambda, which is a well-known characteristic of transonic shock waves. Furthermore, at this step, a trace of a weak shock at the Cone-Parabola junction is seen for the first time.

Within Steps 3 to 6 , by increasing the free-stream Mach number, all the established shock waves get stronger and the transonic characteristics are revealed 
more clearly. The normal shock on junction 4 moves downstream and, as the local Mach number raises, it is extended much more into the flowfield. Evidently, this shock is the most dominant shock wave of the subsonic phase of shock formation process, because it holds all the attributes of transonic shock waves. For the shock at junction 3 , the same trend is seen even though its movement toward downstream is shorter. The formed shock on the conical section seems to be concave with respect to the inflow axis, which is physically impossible. An exact examination points out that this shock is placed normal to the boundary layer on the conical section, that makes it look outwardly with respect to the inflow. As the last point, the tiny shock after junction 1 remains almost unchanged within the subsonic phase steps.

From Steps 7 to 9, different trends are seen with different shocks. For shocks on the cylindrical and parabolic regions (after junctions 3 and 4), the previous trend still goes on. At Step 8, it is completely obvious that the shock on the parabolic section is altered into a lambda one. Further, at this step, the main shock on the cylindrical region is placed exactly on the edge of the test section window and cannot be seen from Step 9 on. In contrast, the strength and extension of the shock on the conical portion decline such that, at Step 9, its size decreases significantly. Due to the very small size of the shock in junction 1 , it is very difficult to follow its variations, although at Step 9, a reduction in its extent is observable.

As the free-stream Mach number approaches unity, the well-known transonic characteristics of the flowfield disappear. This process begins at Step 10, when the normal shock on the parabolic region decreases in extension and strength while gradually returning back to its initial position. At this step, the remains of both junctions of shocks 1 and 2 are infinitesimal. Unfortunately, the window frame limitation hampers us from following the main shock over model A; however, according to the literature, it finally stands on the trailing edge $[5,11]$. Finally, at Step 11, there is no trace of the previous shocks at junctions 1 and 2 and the normal shock on the parabolic portion, which has now moved back exactly on junction 3, is miniature. Now, the shock waves formation process is on the verge of its second phase. By disappearance of all the transonic shocks at Step 12 and emergence of a strong normal shock in front of the model, the second phase or the supersonic phase starts.

\subsection{Supersonic phase}

The flow structure of supersonic phase is much more complex and, since no rigorous research has been conducted to study it, the flow features are mostly unknown and unidentified. To cope with this deficiency, the supersonic process is investigated comprehensively

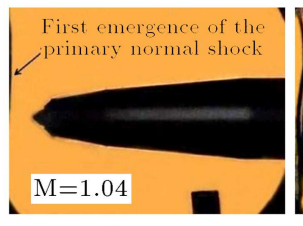

Step 12
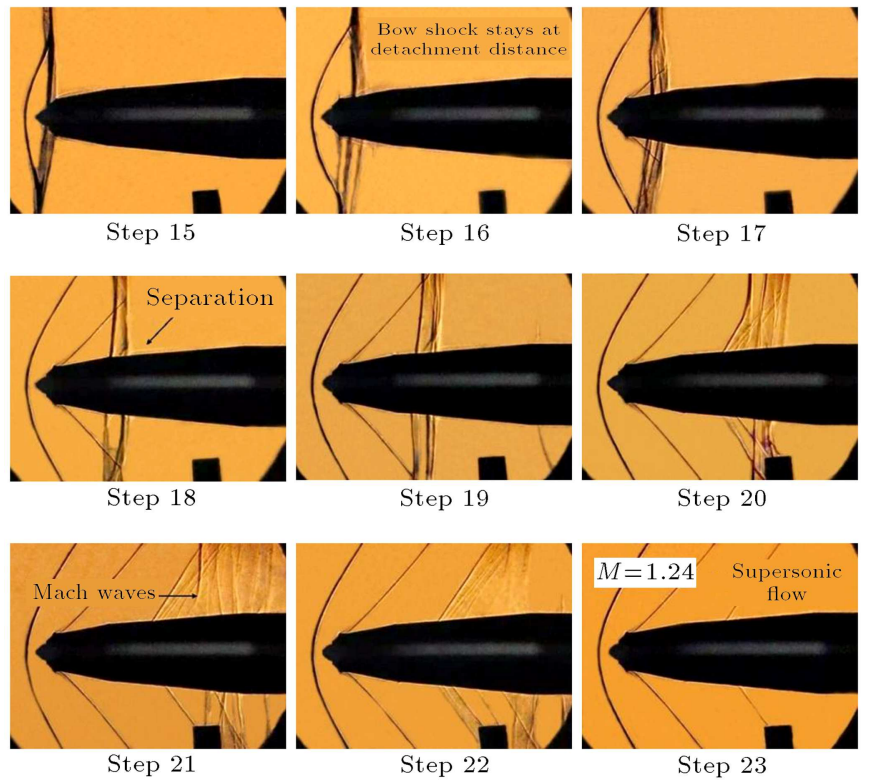

Figure 3. Shadowgraphic visualization of the supersonic phase around model A.

by exploring the shadowgraph results of all three models in details. Furthermore, the authors have designated some patterns and features of the flowfield to organize the process and build up a basis for further studies on this subject.

In Figure 3, a chain of shadowgraphs depicts the supersonic phase of the shock waves formation around model A. The photos are labeled from Steps 12 to 23 in order to keep the overall process, from subsonic to supersonic, in a straight line and to avoid any confusion. Clearly, Step 12 is of the same subsonic phase, emphasizing the process continuity. At this step, the sign of a normal shock is evident, even though, due to the frame dimensions, nothing more is identifiable. Considering this step as the beginning of the supersonic phase can be illusive. Indeed, the initial position of the primarily formed normal shock when the free-stream Mach number reaches unity can be at a farther distance from the model (theoretically at infinity [12]), and by increasing the free-stream Mach number, it approaches the model. In effect, this step is not the first emergence of the normal shock at supersonic phase, even though the time difference is negligible.

The shadowgraph Step 13 discloses the unique phenomenon of supersonic phase of shock system formation, i.e. splitting. The division of a stronger shock wave into two or more weaker shocks is titled 
"splitting" in this paper. It seems that splitting process happens because the flow should become subsonic and, since the primary shock waves are not strong enough to decelerate the flow, a secondary shock wave forms right behind it. The secondary shock(s) assures deceleration of the flow back into subsonic speed. In this regard, splitting plays a major role in reshaping the flowfield structure.

At Step 13, the primary normal shock is split into two adjacent shock waves. These shocks come closer to the model as the free-stream Mach number increases. Both shocks are distorted at their upper ends; this distortion is not an intrinsic trend of the shocks and is allegedly because of some discontinuity on the upper wall of the test section. The convex shape of the farther shock reveals that it is going to establish the supersonic bow shock. The slide of Step 14 depicts this more clearly, where the two adjacent shocks get separated more and more and the establishment of the bow shock is obvious.

A special nomenclature is suggested to describe the unique structure of the shocks. The two adjacent shocks look like a bow and a "Zeh" (Zeh is the exclusive word in Persian literature for the bow string and it is more appropriate to be used here). The convex-shape shock ultimately becomes bow shock, and the straight one is named Zeh shock. The Zeh shock is responsible for forming the final pattern of shock system over the model. From now on, as the free-stream Mach number increases, the Zeh shock goes through a series of actions, which alter its nature thoroughly. By increasing the free-stream Mach number, the Zeh shock begins to split into weaker shocks while moving downstream. Note that the Zeh shock has a low-amplitude oscillation and splitting happens rapidly; thus, the shadowgraphs around it are not clear enough to distinguish the split shock waves. At the same time, the bow shock reaches its final position in Step 15 (Figure 3). The interval between the first emergence of the initial normal shock (the onset of the supersonic phase) and the moment the bow shock stands at its eventual point is designated as the approaching phase.

From Step 16 on, the second phase of supersonic phase, i.e. sweeping phase, begins. As the Zeh shock or its split sweeps the model, the flowfield behind it holds the characteristics of the supersonic regime. For instance, at Step 17, by passing the Zeh shock from the Belt-Cone junction, an oblique shock wave emerges right on the junction. A close examination of this shadowgraph points out that, in addition to the oblique shock formation, a weak Mach wave is also formed after the Nose-Belt junction, which seems to coalesce with the oblique shock.

By increasing the free-stream Mach number, the normal shock splits from the primary Zeh shape and moves downstream while splitting into weaker shocks.
The process continues until they are replaced by several Mach waves. The corresponding photos for Steps 20, 21 , and 22 in Figure 3 show how the adjacent Mach waves get separated from each other, distribute over the model, and finally disappear gradually. Indeed, at Step 23, there is no sign of the primary Zeh shock, which means that the shock wave formation process is practically finished.

In contrast to the subsonic phase, the supersonic phase is accompanied by severe interaction between shock waves and boundary layer, which influences flow separation significantly. The separation variations will be discussed briefly. As the Zeh shock reaches the Belt-Cone junction at Steps 14 and 15 (Figure 3), a perceptible increase in the thickness of the separation layer, in comparison with Steps 12 and 13, is observed. At Step 16, as the Zeh shock passes the Belt region, it falls in the middle of separation layer and makes the layer thicker in the region behind the shock by imposing a higher pressure. At Step 17, the Zeh shock splits into several weaker shocks and an oblique shock appears on junction 2; all separation layers ahead and behind the shocks vanish. Note that the flow behind the oblique shock does not separate since it smooths the pressure gradient ahead and behind it. However, as the split shock reaches the parabola region, the flow separates right after the last shock wave. This separation pattern is seen in Steps 18 and 19. Finally, by appearance of Mach waves, within Steps 20 to 23, no extra pressure is imposed on the flowfield; hence, the boundary layer remains attached over all sections of the model.

\subsection{Shadowgraphs of ogive and inlet models}

Figure 4 shows six consecutive shadowgraph photos illustrating the supersonic phase of the transonic regime around the nose of the ogive model (model B). Since the long cylindrical section of the ogive model is tested with two different configurations, its shadowgraphs are discussed separately. At Step 1, the free-stream Mach number reaches near unity and no shock wave is observable within the scope of shadowgraphs. As the

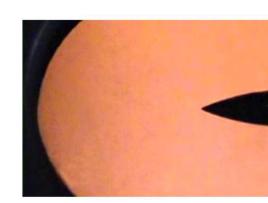

Step 1

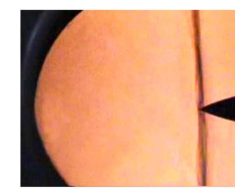

Step 4

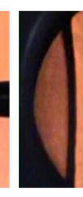

Step 2

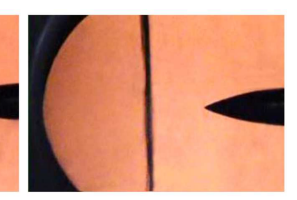

Step 3

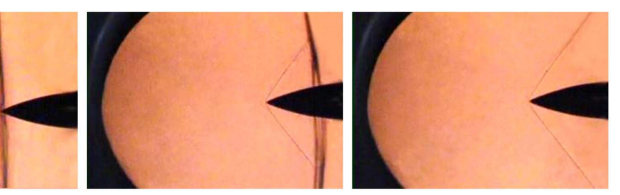

Step 5

Figure 4. Shadowgraphy photographs of supersonic phase around nose of ogive model, model B. 
free-stream Mach number increases, the initial normal shock emerges (Step 2) and approaches the model nose at higher Mach numbers. This normal shock divides the overall flow field into supersonic and subsonic regions (Step 3). In contrast to the previous case, this shock does not split into bow and Zeh ones. This is due to the sharp nose of the ogive model, which prevents the shock to become a bow one. Consequently, the initial normal shock reaches the model intactly and the previous definition of approaching phase becomes inapplicable in Steps 3 and 4. For a pointed body, the approaching phase is defined as the interval between the first emergence of the initial normal shock and the moment it touches the nose of the body.

From Step 4 on, the initial normal shock takes the shape of the so-called Zeh shock, since it passes the model nose while settling down the supersonic flow around model. It must be noted that this nomination is not in disagreement with the previous definition of Zeh shock. Indeed, the normal shock that sweeps the model and splits into weaker shock waves is named the Zeh shock in this paper, irrespective of whether the bow is formed or not. The sweeping phase is completely similar to what was described for model A and begins from Step 4 on. As seen, from Step 5, an attached oblique shock is formed on the nose of model and the Zeh shock seems to move downstream as the free-stream Mach number is further increased. Finally, at Step 6, a full supersonic flow is attained around the nose of the ogive model.

The Zeh shock continues its motion on the cylindrical section of the ogive model. Figures 5 and 6 depict the supersonic phase on this section for both perturbed wire and belt installed configurations, respectively. At Step 1 of Figure 5, a sign of Zeh shock is observable. In addition, a weak oblique shock is seen to form on the wire, which is formed due to the flow expansion behind the trip strip. At Step 2, the Zeh shock sweeps the long body while reducing the local Mach number of the flow field. This is clear due to disappearance of the oblique shock seen in Step 1 (Figure 5). At Step 3, the Zeh shock reaches the middle of the cylindrical section of the model; however, it is not split yet. The first sign of splitting is observed at Step 4, where the Zeh shock has passed the trip strip and a detached oblique shock is established. By increasing the free-stream Mach number, this oblique shock forms in the vicinity of the trip. At this step, i.e. Step 5, the splitting of Zeh shock is clear. Finally, at Step 6, the Zeh shock has swept the body and the characteristics of supersonic regime are settled down. In addition to the detached oblique shock, another shock is formed behind the trip wire, which is clearly due to the expansion wave formed behind the trip strip wire. Note the trip strip wire has a diameter of $0.9 \mathrm{~mm}$ and when it is installed on the model at its present location, the local Reynolds
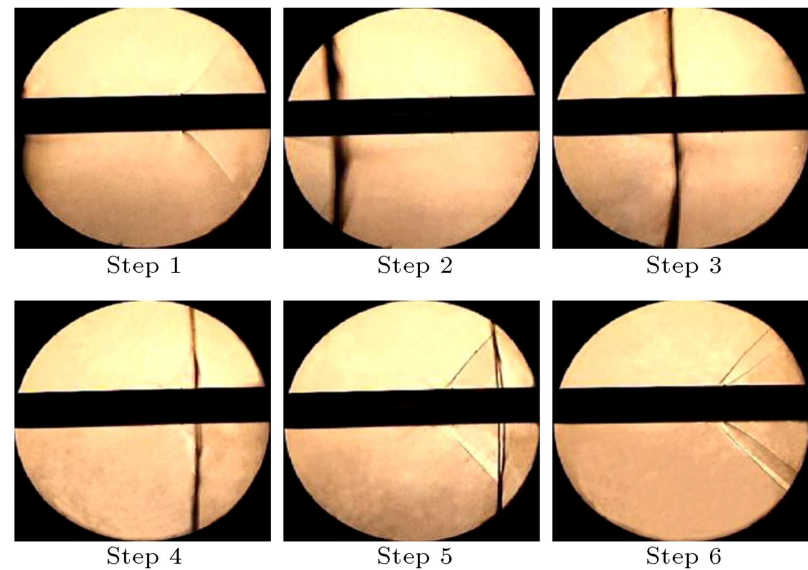

Figure 5. Shadowgraphs of supersonic phase around perturbed cylindrical section of ogive model (with trip strip), model B.
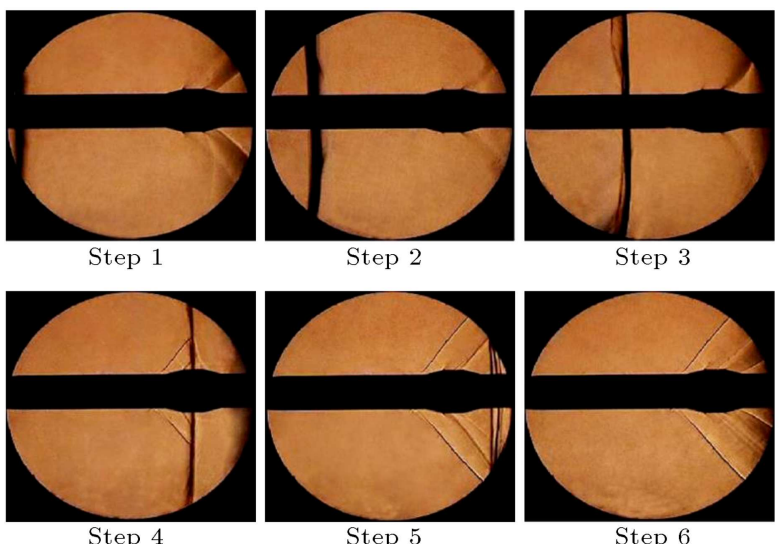

Figure 6. Shadowgraphs of supersonic phase around belt-installed cylindrical section of ogive model, model B.

number over it is equal to $15 \times 10^{6}$, which is enough to perturb the boundary layer.

In Figure 6, the effects of belt on the supersonic phase for model B are investigated. According to the shadowgraphs, in Step 1, the Zeh shock is on the verge of sweeping the long cylindrical section of the model. Further, formation of two consecutive expansion waves is clearly observed that produce a locally supersonic flow and lead to an oblique shock wave. At Step 2, the Zeh shock reaches the belt and, consequently, the expansion waves are weakened and the oblique shock disappears. The same phenomenon is seen in Step 3, where the first expansion wave has nearly disappeared. At Step 4, the Zeh shock is right on the center of the belt and, as expected, a detached oblique shock is formed in front of the belt. Moreover, a secondary oblique shock is established after the first corner of the belt, where an expansion wave is expected; this discrepancy is probably due to some discontinuity on the belt section. At Step 5, this shock seems to be the result of a cluster of recompression waves. At this 
step, the splitting of Zeh shock into two weaker shocks happens. Finally, at Step 6, the shock structure of supersonic flow is settled and a collection of expansion and oblique shock waves establish the flow field around the belt. A complete investigation into flowfield around this model is discussed in [13].

The configuration of inlet model, i.e. model C, is in effect a combination of models $\mathrm{A}$ and $\mathrm{B}$ (from a shock-structure point of view) as an attached oblique shock is established due to the sharp spike and, shortly after it, a bow shock is formed ahead of the inlet entry. This model was designed by Soltani and Farahani to study its performance in supersonic flow; a detailed investigation into this inlet can be found in [14]. In Figure 7, the shadowgraph photos of supersonic phase around this model are exhibited in 9 steps. Analogous to the ogive model, the initial normal shock is shown in Step 1. Obviously, the sharp nose of the spike does not impose a bow shock upstream and the initial normal shock forms on the spike point without splitting. Conclusively, Steps 1 and 2 describe the approaching part of supersonic phase based on the definition that was proposed for the previous ogive model. The sweeping phase begins from Step 3, where the normal shock has passed the spike nose and an attached oblique shock is being formed on the spike. From this step onwards, the initial normal shock is called Zeh shock, as explained previously.

As the Zeh shock passes from the inlet, a bow shock is formed and the flowfield behind it converts into subsonic. The oscillation of this bow shock is a decisive factor in the performance of a supersonic inlet, as its

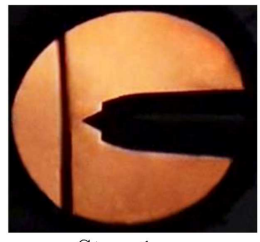

Step 1

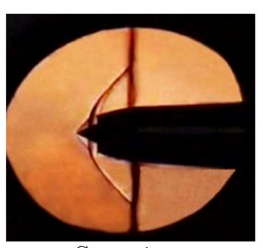

Step 4

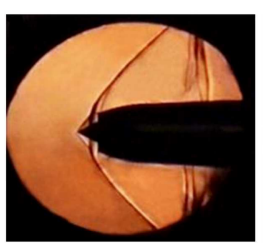

Step 7

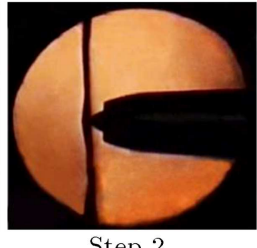

Step 2

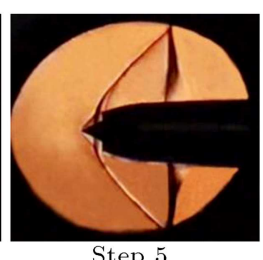

Step 5

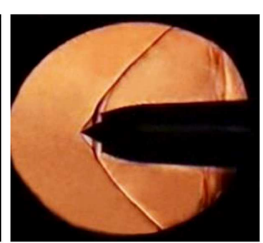

Step 8
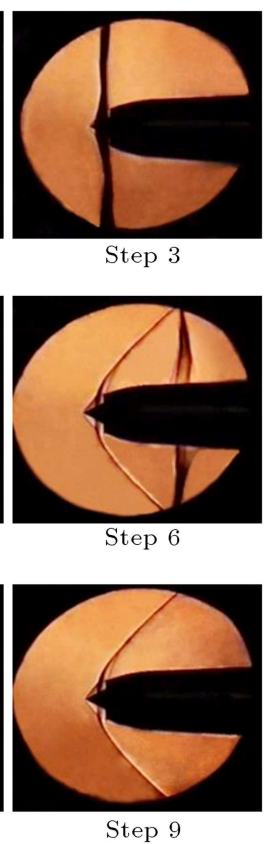

Figure 7. Description of supersonic phase of inlet model by shadowgraphs. oscillation, called 'buzz,' has several detrimental effects on the performance of the propulsion systems [15]. In addition, a compression wave is formed right at the cowl lip that coalesces with the bow shock downstream. From Step 4 on, the Zeh shock over the model sweeps downstream and splits into several weaker shock waves similar to what was seen for models A and B. Photos of Steps 5 and 6 clearly depict the process of transformation of the Zeh normal shock into two weaker ones. At Step 7, several weak normal shocks are seen that change into Mach waves at Step 8. Finally, in the last step, i.e. Step 9, all normal shocks disappear and, consequently, the overall flowfield over the model surface is characterized by the supersonic flow regime.

Now that the main characteristics of the supersonic phase are thoroughly investigated, a general description of the various shock waves is provided. The supersonic phase of the shock wave formation and development process begins with emergence of the initial normal shock wave. For a blunt nose body, the splitting phenomenon divides the initial normal shock into two weaker shocks, a bow, and a Zeh. In contrast, for pointed bodies, the initial normal shock reaches the nose intactly and after that, it is considered as the Zeh shock. By increasing freestream Mach number, the bow shock stops at shock detachment distant, but the Zeh shock keeps moving downstream while persistently being split into weaker shocks. During the sweeping phase, the Zeh shock diminishes completely and, through disappearance of Mach waves, the transonic regime is completed. In Figure 8 all steps of the supersonic phase over a blunt body are categorized and demonstrated schematically.

\subsection{Criterion of transition from transonic to supersonic}

As a rule of thumb, for free-stream Mach numbers in the range of 0.8 to 1.2 , the flow is called transonic. The lower limit of this range is usually in proximity of the critical Mach number and, therefore, it can be representation of the first establishment of shock waves. However, in terms of physical reasoning, the upper limit is ambiguous. It does not imply any specific behavior of the flowfield to indicate the transition from transonic regime to the supersonic one. Seemingly, taking this range as transonic flow is mostly based on a force viewpoint, especially drag variations, which shows strange behaviors in this range. However, the aerodynamic forces result from the flowfield features and any change in their pattern requires a physical explanation.

With regard to the shadowgraphs of the supersonic phase, a physical interpretation is proposed to differentiate the supersonic regime from the transonic one. According to this proposal, the complete disappearance of the Zeh shock, which takes place because 


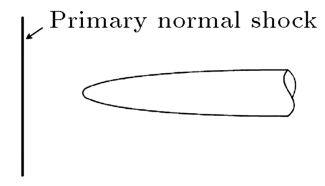

(a) The onset of approaching phase

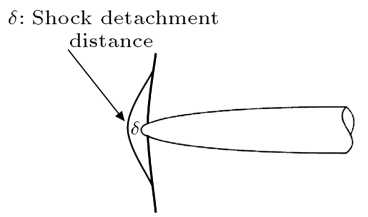

(c) The onset of sweeping phase

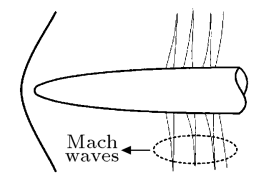

(e) Splitting into Mach waves

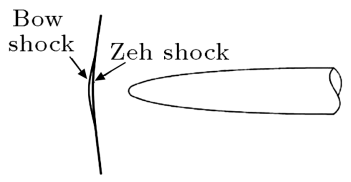

(b) Splitting of initial normal shock

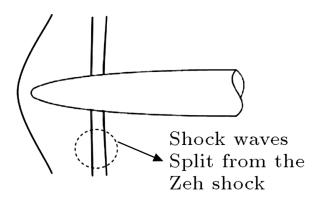

(d) Splitting of Zeh shock

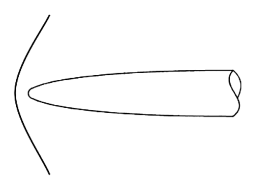

(f) The onset of supersonic regime

Figure 8. A schematic representation of supersonic phase of shock waves formation process over a general blunt body.

of the continuous splitting of shock waves, determines the onset of supersonic regime. In effect, shortly after appearance of Mach waves, the whole flowfield is characterized by the supersonic flow. There is an exception for blunt bodies, where practically a small area of subsonic flow always exists and the theoretical definition of the supersonic regime (A flowfield is called supersonic if Mach number is greater than 1 at every point [13].) is not applicable. However, a compromise is usually made to ignore this subsonic area behind the bow shock, because by increasing the free-stream Mach number, it gets so small that its effects on the flowfield are negligible.

The extreme variations of drag force in transonic flow are well-known and, as was mentioned before, its trends are dictated by the structure of shock waves. Figure 9 depicts a typical variation of drag coefficient versus free-stream Mach number, illustrating the critical and drag-divergence Mach numbers accompanied by shadowgraph photos over the model to provide a physical interpretation. The critical Mach number is concurrent with the first emergence of a sonic flow over the body and, by appearance of a strong shock, the drag coefficient increases, called drag-divergence. As the free-stream Mach number reaches unity, the initial normal shock forms upstream, which results in an intensive amount of energy loss; this explains the maximum point of drag force variations. At Mach numbers higher than unity, the Zeh shock begins to split into weaker normal shocks and is substituted with oblique shocks. This mitigates the negative effect of shock waves and gradually decreases the drag force. At

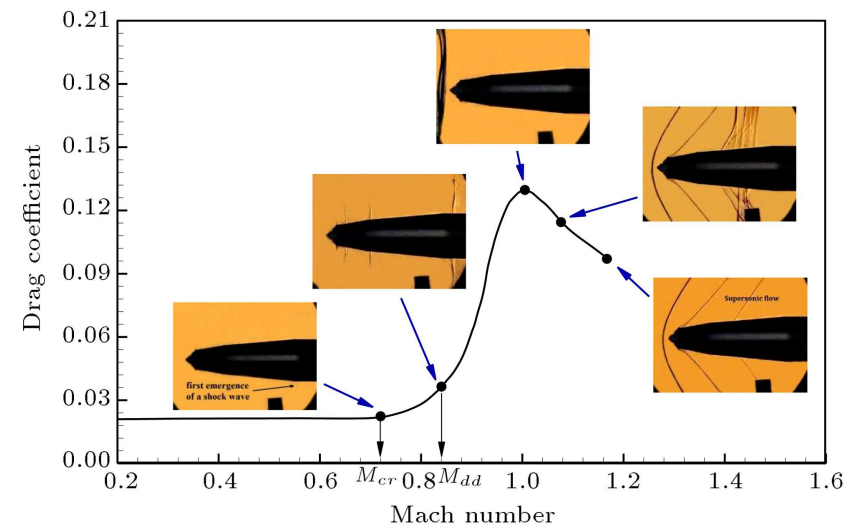

Figure 9. Typical variation of drag coefficient in conjunction with shock waves structure in transonic flow [16].

last, by the end of sweeping phase and disappearance of all normal shocks, less drag force is induced on the body. Based on the body configuration, drag force can increase or decrease at higher Mach numbers; however, this subject is beyond the scope of this investigation at the present time.

\section{Conclusions}

An experimental investigation was conducted to study the shock waves system using shadowgraph visualization technique. While primarily qualitative, this study provides valuable insights into the formation and development process of shock waves. In order to generalize the results, experiments were performed for three different models. The following specific conclusions are attained:

1. The shock waves formation and development process was categorized into two phases, namely, subsonic and supersonic phases. This nomination is based on the free-stream Mach number. The shadowgraphs showed that for the subsonic phase, different shocks appeared over the model A, even though only two of them contained all the known characteristics of transonic shock waves;

2. What is normally known as the transonic regime is usually referred to as the subsonic phase and the supersonic one is almost ignored. The supersonic phase consists of two consecutive phases itself, namely, "approaching phase" and "sweeping phase." The definitions of approaching phase for blunt and sharp nose bodies were found to be different;

3. Splitting turned out to be the principal phenomenon of supersonic phase, which formed the ultimate structure of shock waves. This process proceeded forward within the sweeping phase until the Zeh shock diminished completely and the tran- 
sonic regime ended. Moreover, at supersonic phase, the interaction between shock waves and boundary layer became severe;

4. A physical interpretation was proposed to distinguish between transonic and supersonic regimes. According to this definition, the complete disappearance of Zeh shock that happens because of splitting is the key criterion of this transition. By this explanation, the extreme trends of drag force in transonic flow were clarified in terms of physical reasoning.

\section{Acknowledgment}

This work was funded partly by Sharif University of Space Technology and Transportation Research Institute, Iranian Space Research Center. Their support is gratefully acknowledged. The authors wish to thank the service rendered by the staff of Qadr Aerodynamic Research Institute for carrying out all tests.

\section{References}

1. Babinsky, H. and Harvey, J.K., Shock Wave-BoundaryLayer Interactions, 20, Cambridge University Press (2011).

2. Olivier, H., Reichel, T. and Zechner, M. "Airfoil flow visualization and pressure measurements in highReynolds number transonic flow", AIAA Journal, 41(8), pp. 1405-1412 (2003).

3. McDevitt, J.B., Levy Jr., L.L. and Deiwert, G.S. "Transonic flow about a thick circular-arc airfoil", AIA A Journal, 14(5), pp. 606-613 (1976).

4. Mabey, D.G. "Physical phenomena associated with unsteady transonic flows", AIA A Journal, 120, Washington, pp. 1-55 (1989).

5. Pearcy, H.H. "Some effects of shock-induced separation of turbulent boundary layers", in Transonic Flow Past Aerofoils, HM Stationery Office (1959).

6. Liepmann, H.W. "The interaction between boundary layer and shock waves in transonic flow", Journal of the Aeronautical Sciences, 13(12), pp. 623-637 (1946).

7. Patz, G. "Formation of bow waves around blunt bodies in the flow behind a moving shock", Acta Mechanica, 32(1-3), pp. 89-100 (1979).

8. Davies, L., Bow-Shock Establishment and StagnationPoint Pressure Measurement for a Blunt-Nosed Body at Supersonic Speeds, HM Stationery Office (1965).
9. Andrews, C.D., Carlson, D.R. and Marshall, G.C, "Shadowgraphy study of the upper stage flow fields of some Saturn V study configurations in the transonic Mach number range", NASA TN D-2755, Washington, D.C. (1965).

10. Soltani, M.R., Taeibi-Rahni, M., Farahani, M., et al. "Flow measurements around a long axisymmetric body with varying cross section", 43rd AIAA Aerospace Sciences Meeting and Exhibit (2005).

11. Seegmiller, H.L., Marvin, J.G. and Levy Jr., L.L. "Steady and unsteady transonic flow", AIAA Journal, 16(12), pp. 1262-1270 (1978).

12. Ramm, H.J., Fluid Dynamics for the Study of Transonic Flow, Oxford University Press (1990).

13. Heidari, M.R., Farahani, M., Soltani, M.R. and TaeibiRahni, M. "Investigations of supersonic flow around a long axisymmetric body", Scientia Iranica, 16(6), pp. 534-544 (2009).

14. Soltani, M.R. and Farahani, M. "Performance study of an inlet in supersonic flow", Proceedings of the Institution of Mechanical Engineers, Part G: Journal of Aerospace Engineering, 227(1), pp. 159-174 (2012).

15. Soltani, M.R., Younsi, J.S., Farahani, M. and Masoud, A. "Numerical simulation and parametric study of a supersonic intake", Proceedings of the Institution of Mechanical Engineers, Part G: Journal of Aerospace Engineering, 227(3), pp. 467-479 (2012).

16. Anderson Jr, J.D., Fundamentals of Aerodynamics, Fifth Edition, McGraw-Hill Education (2010).

\section{Biographies}

Mohammad Farahani is an Assistant Professor in the Aerospace Engineering Department of Sharif University of Technology, Tehran, Iran. He has a PhD in Propulsion from Sharif University of Technology. His research interests include applied aerodynamics, unsteady aerodynamics, wind tunnel testing, gas dynamics, and turbo machinery.

Amin Jaberi earned his BS degree in Aerospace Engineering from Malek-e-Ashtar University of Technology and MS degree in the same subject from Sharif University of Technology. His main research interests are experimental aerodynamics, wind tunnel testing, and aircraft design. 